Aus dem Chemischen und Bakteriologischen Laboratorium von Dr. Aufrecht in Berlin.

\title{
Ueber Nährwert und Ausnutzung roher und weichgekochter Hühnereier.
}

Von Dr. S. Aufreclst und Dr. Fr. Simon.

Unter den "Volksnahrungsmitteln" nehmen die Vogeleier einen recht bescheidenen Platz ein. Näheren AufschluB über diese Verhältnisse gibt eine umfangreiche Statistik, die auf die Ernährung von 2567 amerikanischen Arbeiterfamilien Bezug nimmt. Nach diesen Erhebungen (1) kamen von den Kalorien, die von der einzelnen Arbeiterfamilie für ihre Ernährung ver. wendet wurden, durchschnittlich auf

$$
\begin{aligned}
& \text { Fleisch, Geflügel, Fisch . . . . } 24 \% \\
& \text { Eier . . . . . 2\% } \\
& \text { Milch, Käse, Butter, Speck : . . } 22 \% \\
& \text { Zucker und Sirup . . . . . . . } 12 \% \\
& \text { Brot, Mehl, Kartoffeln, Reis . . . . } 40 \%
\end{aligned}
$$

Die Verteilung der Kalorien in dieser Kost, bei der $48 \%$ des Brennwertes durch Nahrungsmittel animalischen Ursprunges gedeckt werden, dürfte in analoger Weise wohl in Deutschland nur bei den wohlhabenden Klassen zum Ausdruck kommen; und man wird nicht fehl gehen, den Anteil, der den Eiern in der durchschnittlichen Ernährung unserer Arbeiterbevölkerung zukommt, vielleicht noch niedriger als mit $2 \%$ zu veranschlagen. Gleichwohl haben die Vogeleier und insbesondere die Hühnereier in der Krankenkost immer eine reichliche und mannigfaltige Verwendung gefunden. Diätverordnungen, die den Genub von Eiern und Eierspeisen vorschreiben, pllegen von den meisten Patienten gewissenhaft, wenn auch vielleicht bisweilen widerwillig ausgeführt zu werden, wie sich ja überhaupt die Eier bezüglich ihrer Nährkraft und Verdaulichkeit beim Publikum einer großen und oft übertriebenen Wertschätzung erfreuen. So erscheint es auffallend, daß verhältnismäßig wenige Arbeiten vorliegen, die sich mit der Magen- und Darmverdaulichkeit der Vogeleier, mit ihrem Nährwert und ihrer Ausnutzbarkeit im menschlichen Organismus beschäftigen.

Von den Arbeiten, die die Magenverdaulichkeit der Eier zum Gegenstand haben, kommen aus der älteren Literatur zunächst die Untersuchungen in Betracht, die Uffelmann (2) im Jahre 1877 an einem gastrotomierten Knaben angestellt hat. Das Untersuchungsmaterial wurde in der Weise gewonnen, daß der Patient flüssige und feste Speisen zunächst im Munde verarbeitete, durchkaute und einspeichelte und dann durch einen in die Fistel mündenden Gummischlauch in den Magen entleerte. Soweit nun Uffelmanns Beobachtungen unser Thema betreffen, lassen sich seine Feststellungen dahin zuzammenfassen, daß bei Verabreichung "nicht weich gekochter" Eier in den Faeces massenhaft Eistückchen mit ganz glatter Oberfläch $\theta$ wiedergefunden wurden, daß 2 Stunden nach Verlütterung weichgekochter Eier sich im Magen noch Reste von „ungemein cohärentem Weißei“ nachweisen ließen. Dagegen konnten bei Einführung von Eiweißwasser in den nüchternen Magen schon nach 20 Minuten Peptone nachgewiesen werden. Allerdings wird man irgendwelche Schlüsse aus Uffelmanns Untersuchungen nur mit Vorsicht und unter Beobachtung des Umstandes ziehen dürfen, daß die Versuchsperson fast während der ganzen Beobachtungszeit fieberte. Viel wertvoller sind die Ergebnisse, die wir den Untersuchungen Penzoldts (3) und seines Schülers Prager (4) verdanken. Diese Autoren ermittelten die Aufenthaltsdauer der Eier (in verschiedener Zubereitung) im Magen des gesunden Menschen durch Magenausheberung. Es stellten sich dabei als Aufenthaltsdauer im Magen folgende Zeiten heraus:

Für 100,0 g Eier (3 Minuten in siedendem Wasser) 1 Std. $45 \mathrm{Min}$.

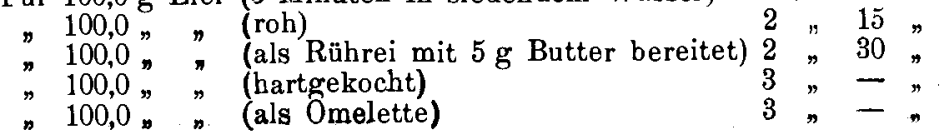


Wenn es erlaubt ist, von der Aufenthaltsdauer der Speisen im Magen auf ihre Verdaulichkeit zu schliefen, so würden unter den Eierspeisen die weichgekochten Eier als die am leichtesten verdaulichen zu gelten haben. An zweiter Stelle würden die rohen Eier und die (als Rührei) mit Butter zubereiteten stehen, während man den hartgekochten Eiern und dem Eierauflauf das geringste Maß von Verdaulichkeit zuerkennen müßte.

Doch alle experimentellen Daten über die Magenverdaulichkeit eines Nährungsmittels vermögen keinen Aufschluß über seinen wirklichen Nährwert zu geben.

Mit vollem Recht sagt daher Rubner (5): „Ueber die Ausnutzbarkeit kann nur das physiologische Experiment am Menschen selbst entscheiden. Experimente über die Magenverdauung allein oder gar die Prüfung mit künstlichen Verdauungssäften geben keinen Einblick in die Verbältnisse." Von diesen Gesichtspunkten aus muß auch eine schon aus dem Jahre 1842 stammende Arbeit von C. G. Lehmann (6) für unseren Gegenstand gewürdigt werden.

Dieser Forscher studierte die Zusammensetzung des Harnes bei verschiedener Kost, wobei er an sich selbst experimentierte. Bei seinen Untersuchungen über den Einfluß der Eierkost auf die Veränderungen des Harnes nahm er an vier aufeinander folgenden Tagen zusammen 128 Hühnereier auf. Auf Grund seiner Analysen berechnete er den Gehalt der täglichen Eierration an "trockenem aschefreiem Albumin " auf 189,7 g, die 30,35 g Stickstoff entsprechen würden. Es wurden täglich durchschnittlich 54,772 g Harnstoff (entsprechend $25,56 \mathrm{~g} \mathrm{~N}$ ) ausgeschieden. Von dem eingeführten Stickstoff wurden also täglich durchschnittlich 84,22\% (als Harnstoff) durch den Harn wieder abgegeben. Von ungleich größerem Wert für die Beurteilung der Ausnutzbarkeit eines Nahrungsmittels sind solche Stoffwechselversuche, die nicht nur die Ausscheidungen in Harn, sondern auch die mit den Faeces abgegebenen Mengen an Trockensubstanz, Stickstoff und Fett berücksichtigen. Von den Untersuchungen, die nach diesen Grundsätzen ausgeführt wurden, kommen in erster Reihe wohl die klassischen Arbeiten Rubners und für unseren Gegenstand seine 1879 publizierten (7) Ausnutzungsversuche in Betracht. Hier berichtet $R u b n e r$ u. a. über einen Versuch, den er an einem 24 Jahre alten, $46 \mathrm{~kg}$ schweren Studenten anstellte. Die Versuchsperson verzehrte an zwei aufeinander folgenden Tagen zusummen 42 Stück hartgekochte Eier, die einem Gewicht von $189 \hat{2}, 2 \mathrm{~g}$ mit einem N-Gehalt von $41,5 \mathrm{~g}$ und einem Fettgehalt von $206.7 \mathrm{~g}$ entsprachen. Die Menge des an beiden Versuchstagen durch Harn und Kot ausgeschiedenen Stickstoffes betrug $45,0 \mathrm{~g}$. Der Körper hatte also während des Eierernährungsversuches $3,5 \mathrm{~g}$ Stickstoff oder $21,88 \mathrm{~g}$ Eiweiß verloren. Der prozentige Verlust an den einzelnen Bestandteilen der Eier und des Fleisches ${ }^{1}$ ) durch den Kot betrug:

\begin{tabular}{|c|c|c|}
\hline & für Eier & für Fleisc \\
\hline ickst & 5,2 & $\left.(5,3)^{2}\right)$ \\
\hline & $\begin{array}{ll}\left.(4,4)^{2}\right) & 5,0\end{array}$ & \\
\hline
\end{tabular}

Die Versuche Rubners an gesunden Menschen ergaben also das Resultat, daß hartgekochte Eier mit Bezug auf die Ausnutzbarkeit ihrer Trockensubstanz und ihres Stickstoffes dem gebratenen Fleische fast gleich zu setzen waren. Die Erscheinung, daß das Fett der harten Eier entschieden besser resorbiert wurde als das des gebratenen Fleisches, erklärt Rubner durch den Umstand, daß in den Eiern absolut viel mehr Fett verzehrt worden war. Aus neuester Zeit endlich liegt noch eine Arbeit von Lebbin (8) vor, der die Ausnutzbarkeit der Eier an einem 28 Jahre alten und $65 \mathrm{~kg}$ schweren Diener prïfte. Die Versuchsperson verzehrte an zwei aufeinander folgenden Tagen 22 (5 Minuten gekochte) Eier, die $1062,3 \mathrm{~g}$ Eisubstanz entsprachen. Auf Grund seiner Kotanalysen berechnet Le b bin den prozentigen Verlust an Eiern

$$
\begin{array}{ll}
\text { an Trockensubstanz auf } & 4,99 \% \\
\text { an Protein auf } & 2,41 \% \\
\text { an Gesamt-Aetherextrakt auf } & 4,23 \%
\end{array}
$$

Diese Zahlen stimmen mit den Resultaten Rubners annährend überein.

Bei allen diesen Stoffwechselversuchen, die sich auf die Ausnutzbarkeit der Eier bezogen, war mehrere Tage hindurch das gleiche Nahrungsmittel ausschlieblich und in solcher Menge zur Aufnahme gekommen, dab dadurch allein schon eine annähernde Deckung des täglichen Bedarfes erzielt wurde.

Diese Versuchsanordnung war auf der wohlberechtigten Erwägung basiert, daß der von den Verdauungssäften gelieferte und mit dem Kot zur Ausscheidung kommende Anteil an

1) Aus zwei anderen Versuchen Rubners als Mittelwert berechnet und der Vergleichung wegen daneben aufgeführt. - 2) Die eingeklammerten Zahlangaben finden sich in Rub ners Lehrbuch der Hygiene 1907, S. 476.
Stickstoff, Fett etc. umso geringer im Verhältnis zur Menge des gesamten Kotstickstoffes, Kotfettes etc. ausfällt, je relativ bedeutender der Stickstoff und Fettgehalt der verabreichten Kost bemessen wird. Um also ein möglichst getreues Bild der Ausnutzbarkeit der Eier zu gewinnen und um zu vermeiden, daß die Resorptionsgröße der Eier mit einem hinter ihrem wirklichen Werte zurückbleibenden veranschlagt werde, mußten absolut recht große Mengen von Eiern verzehrt werden. So grobes theoretisches Interesse aber auch derartige Untersuchungen bieten mögen, so läßt sich doch nicht verkennen, daß Kostordnungen, bei denen täglich ausschließlich 10, 20, 30 Eier verzehrt werden müssen, von den wirklichen $\mathrm{Be}$ dingungen einer rationellen Gesunden- und Krankenernährung recht erheblich abweichen. Wir hatten uns deshalb die Aufgabe gestellt, die Ausnutzbarkeit der Eier als Bestandteil einer gemiscliten Kost neben anderen, gleichzeitig gereichten Nahrungsmitteln zu prüfen. Selbstverständlich mußte die Quote der anderen, neben den Eiern gereichten Nahrungsmittel in bezug auf Beschaffenheit, Menge und Zubereitung stets konstant gehalten werden. Als Vorperiode wählten wir eine Kost, bei der - ceteris paribus - die Eier durch entsprechende Mengen von Fleisch ersetzt wurden. Ferner erschien es uns von Wichtigkeit, bei unseren Ausnutzungsund Stoffwechselversuchen auch das Verhalten der rohen Eier neben dem der weichen besonders zu prüfen, da die Untersuchungen Rubners nur auf hartgekochte, die Lebbins nur auf halbharte Rücksicht nehmen, somit genauere Ausnutzungsversuche der rohen und ganz weichen Eier bisher nicht vorliegen.

Die Versuchsanordnung gestaltete sich also derart, daß der eine von uns (A., 44 Jahre alt, $84 \mathrm{~kg}$ schwer) während der ganzen Beobachtungsdauer als Versuchsperson diente, ohne dabei die gewohnte Lebensweise und Tätigkeit zu verändern. Die ganze Versuchszeit, die sich über $7 \mathrm{mal}$ 24 Stunden in ununterbrochener Folge erstreckte, wurde in drei Perioden geteilt. Die erste Periode umfaßte drei Tage. Während dieser Periode bestand die tägliche Kost aus: Kaffee, Zucker, Weißbrot, Butter, Schweineschmalz, Reissuppe, Apfelkompott, gesottenen Kartoffeln, Beefsteak, geräucherter Ochsenzunge und Schinken. Als Getränk diente Moselwein und Zitronenlimonade. Die zweite Periode umfaßte 2 Tage. Während dieser Periode wurden - - mit Ausnahme der Fleischspeisen - genau die gleichen Nahrungsmittel in derselben Beschaffenheit, Zubereitung und Menge, wie sie während der ersten Periode gereicht worden waren, aufgenommen. An die Stelle der Fleischspeisen der ersten Periode (150 g Fleisch mit $43,65 \mathrm{~g}$ Stickstoffsubstanz und $29,85 \mathrm{~g}$ Fett) traten aber in der zweiten Periode 8 große, 3 Minuten in siedendem Wasser behandelte und von der Schale befreite Hühnereier, die zusammen $415 \mathrm{~g}$ Eisubstanz mit 52,29 g Stickstoffsubstanz und $49,8 \mathrm{~g}$ Fett entsprachen. In der dritten Periode, die sich über zwei Tage erstreckte, setzte sich die Kost aus den bei der zweiten Periode angeführten Nahrungsmitteln in den genau entsprechenden Mengenverhältnissen zusammen, mit der einzigen Abänderung, daß an Stelle der weichgekochten Eier die gleichen Mengen roher Eier genossen wurden.

Stickstoff- und Fettbilanz während der ersten Periode. $\mathrm{T}$ abelle $\mathrm{l}$.

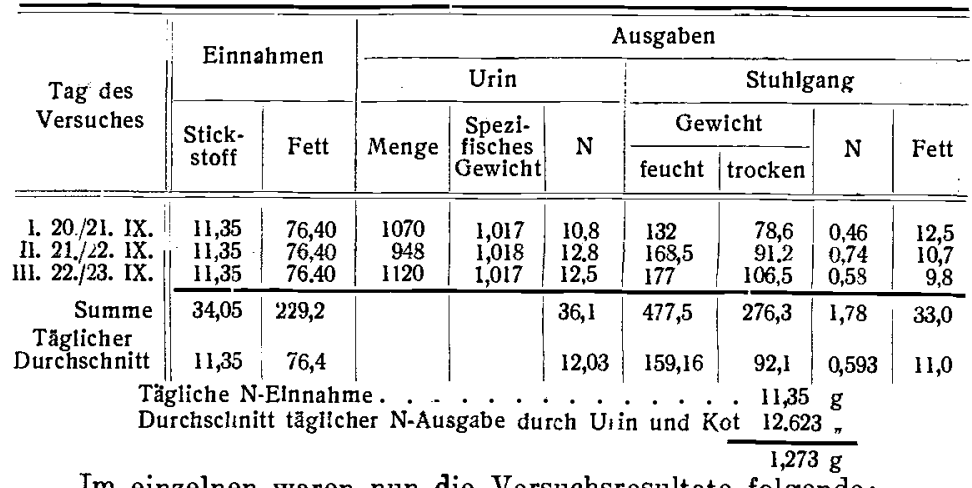

Im einzelnen waren nun die Versuchsresultate folgende:

Erste Periode, umfassend drei Tage (20./21., 21./22. 22./23. September 1908). Die Kost bestand aus: $200 \mathrm{~g}$ Kaffee, $30 \mathrm{~g}$ Zucker, $200 \mathrm{~g}$ Weißibrot, $25 \mathrm{~g}$ Butter, $25 \mathrm{~g}$ Schweineschmalz, 
$150 \mathrm{~g}$ Reissuppe, $50 \mathrm{~g}$ Apfelkompott, $100 \mathrm{~g}$ gesottenen Kartoffeln, $100 \mathrm{~g}$ Beefsteak, $25 \mathrm{~g}$ geräucherter Zunge, $25 \mathrm{~g}$ Schinken. Mit diesen Nahrungsmitteln wurden täglich aufgenommen: $70.92 \mathrm{~g}$ Stickstoffsubstanz $(=11,35 \mathrm{~g} \mathrm{~N}), 76,4 \mathrm{~g}$ Fett und $245,9 \mathrm{~g}$ Kohlehydrate. Summa der täglich eingeführten Kalorien $=2013,7$.

Der Körper gab also während der ersten Periode täglich durchschnittlich $1,273 \mathrm{~g} \mathrm{~N}=7,956 \mathrm{~g}$ Eiweiß ab.

Von dem eingeführten Stickstoff wurden durch den Kot täg. lich durchschnittlich $5,22 \%$ wieder abgegeben.

Von dem eingeführten Fett wurden durch den Kot täglich durchschnittlich $14,39 \%$ wieder abgegeben.

Zweite Periode, umfassend zwei Tage (23./24. und 24./25. September 1908). Die Kost bestand aus: $200 \mathrm{~g}$ Kaffee, $30 \mathrm{~g}$ Zucker, $200 \mathrm{~g}$ Weißbrot und 8 großen, weichgekochten Eiern (entsprechend $415 \mathrm{~g}$ Eisubstanz). Mit diesen Nahrungsmitteln wurden täglich aufgenommen : 79,56 $\mathrm{g}$ Stickstoffsubstanz ( $=12,73 \mathrm{~g} \mathrm{~N})$, $96,35 \mathrm{~g}$ Fett und $245.9 \mathrm{~g}$ Kohlehydrate. Summe der täglich eingeführten Kalorien $=2230.6$.

Stickstoff- und Fettbilanz wăhrend der zweiten Periode. Tabel1e 2 .

\begin{tabular}{|c|c|c|c|c|c|c|c|c|c|}
\hline \multirow{4}{*}{$\begin{array}{l}\text { Tag des } \\
\text { Versuches }\end{array}$} & \multirow{2}{*}{\multicolumn{2}{|c|}{ Einnahmen }} & \multicolumn{7}{|c|}{ Ausgaben } \\
\hline & & & \multicolumn{3}{|c|}{ Urin } & \multicolumn{4}{|c|}{ Stuhlgang } \\
\hline & \multirow{2}{*}{$\begin{array}{l}\text { Stick- } \\
\text { stoff }\end{array}$} & \multirow{2}{*}{ Fett } & \multirow{2}{*}{ Menge } & \multirow{2}{*}{$\begin{array}{l}\text { Spezi- } \\
\text { fisches } \\
\text { Gewicht }\end{array}$} & \multirow{2}{*}{ N } & \multicolumn{2}{|c|}{ Gewicht } & \multirow{2}{*}{$\mathrm{N}$} & \multirow{2}{*}{$F_{t} t t$} \\
\hline & & & & & & feucht & trocken & & \\
\hline IV. $23 / 24$. IX. & 12,73 & 96,35 & 1270 & $\mathrm{I}, 028$ & 13,90 & 154,2 & 94,7 & 0,42 & 5,70 \\
\hline V. $24 . / 25$. IX. & 12,73 & 96,35 & 1160 & 1,027 & 13,66 & 206 & 102 & 0,55 & 6,35 \\
\hline Summe & $\overline{25,46}$ & 192,7 & & & 27,56 & 360,2 & 196,7 & 0,97 & 12,05 \\
\hline $\begin{array}{l}\text { Täglicher } \\
\text { Durchschnitt }\end{array}$ & 12,73 & 96,35 & & & 13,78 & $\mathrm{I} 80, \mathrm{I}$ & 98,35 & 0,485 & 6,025 \\
\hline
\end{tabular}

Der Körper gab also während der zweiten Periode täglich durchschnittlich 1,535 g $\mathrm{N}=9,594$ g Eiweiß ab.

Von dem eingeführten Stickstoff wurden durch den Kot täglich durchschnittlich $3.81 \%$ wieder abgegeben.

Von dem eingeführten Fett wurden durch den Kot täglich durchschnittlich $6,25 \%$ wieder abgegeben.

Dritte Periode, umfassend zwei Tage (25./26. und 26./27. September 1908). Die Kost bestand aus: $200 \mathrm{~g}$ Kaffee, $30 \mathrm{~g}$ Zucker, $200 \mathrm{~g}$ Weißbrot, $25 \mathrm{~g}$ Butter, $25 \mathrm{~g}$ Schweineschmalz $150 \mathrm{~g}$ Reissuppe, $50 \mathrm{~g}$ Apfelkompott, $100 \mathrm{~g}$ gesottenen Kartoffeln und 8 großen, rohen Eiern (entsprechend $415 \mathrm{~g}$ Eisubstanz). Mit diesen Nahrungsmitteln wurden täglich aufgenommen: 79,56 g Stickstoffsubstanz ( $=12,73 \mathrm{~g} \mathrm{~N}$ ), 96,35 $\mathrm{g}$ Fett und $245,9 \mathrm{~g}$ Kohlehydrate. Summe der täglich eingeführten Kalorien $=2230,6$.

Ta belle 3 .

Stickstoff- und Fettbilanz während der dritten Periode.

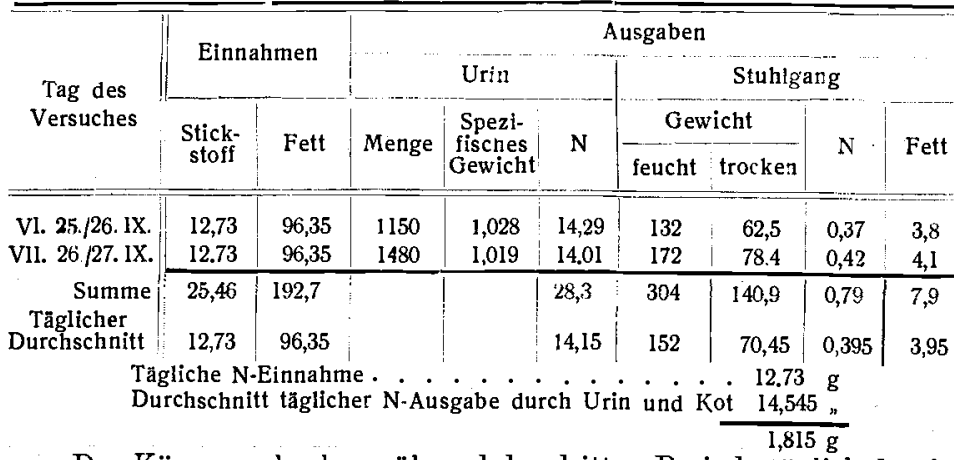

Der Körper gab also während der dritten Periode täglich durchschnittlich $1,815 \mathrm{~g} \mathrm{~N}=11,344 \mathrm{~g}$ Eiweiß ab.

Von dem eingeführten Stickstoff wurden durch den Kot täglich durchschnittlich $3,1 \%$ wieder abgegeben.

Von dem eingeführten Fett wurden durch den Kot täglich durchschnittlich $4,09 \%$ wieder abgegeben.

$$
\text { Tabell e } 4 .
$$

Vergleichende Tabelle der drei Versuchsperioden.

\begin{tabular}{|c|c|c|c|c|c|}
\hline \multirow[b]{2}{*}{ Periode } & \multirow{2}{*}{ 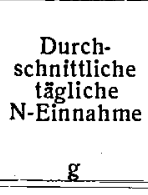 } & \multirow{2}{*}{$\begin{array}{c}\text { Durch- } \\
\text { schnittlicher } \\
\text { tăglicher } \\
\text { N-Verlust } \\
\mathrm{g} \\
\end{array}$} & \multicolumn{2}{|c|}{$\begin{array}{c}\text { Es wurden nicht resor- } \\
\text { biert in } \%\end{array}$} & \multirow[b]{2}{*}{ Bemerkungen. } \\
\hline & & & $\begin{array}{c}\text { vom ein- } \\
\text { geführten N } \\
\% \\
\end{array}$ & $\begin{array}{l}\text { vom ein- } \\
\text { geführten } \\
\text { Fett } \\
\%\end{array}$ & \\
\hline I & $\begin{array}{l}\mathrm{II}, 35 \\
\mathrm{I} 2,73\end{array}$ & $\begin{array}{l}1,273 \\
1,535\end{array}$ & $\begin{array}{l}5,22 \\
3,81\end{array}$ & $\begin{array}{r}\mathrm{I} 4,39 \\
6.25\end{array}$ & $\begin{array}{l}\text { Fleischperiode. } \\
\text { Periode der weich- }\end{array}$ \\
\hline III & 12,73 & 1,815 & 3,10 & 4,09 & $\begin{array}{l}\text { Periode der rohen } \\
\text { Eier. }\end{array}$ \\
\hline
\end{tabular}

Schliusse. Aus den gegenseitigen Beziehungen der Versuchsresultate aller drei Perioden ergibt sich nun zunächst, daß die Größe des durchschnittlichen täglichen Stickstoffverlustes proportional der Menge des resorbierten, nicht etwa des aufgenommenen Stickstoffes stieg, ein Verhalten, das mit den bekannten Gesetzen der gesteigerten Eiweibzersetzung bei vermehrter Eiweißresorption übereinstimmt. Ferner zeigte sich bei unseren Versuchen, dab sowohl in der Fleischperiode als auch in beiden Eierperioden die Ausnutzung des eingeführten Stickstoffes besser war als die des eingeführten Fettes, in Analogie der bereits von Rubner festgestellten Tatsachen, wenn es überhaupt angängig ist, die mit einer abweichenden Versuchsanordnung erzielten Ergebnisse Rubners zum Vergleiche heranzuziehen. Was nun die Resorptionsgröße des Stickstoffes und Fettes in den einzelnen Versuchsperioden betrifft, so erwies sie sich für beide als die geringste in der Fleischperiode. Die Resorptionsgröbe des Stickstoffes und Fettes stieg dann in der Periode der weichgekochten Eier, um in der Periode der rohen Eier noch weiter, wenn auch unbedeutend zuzunehmen. Wenn wir (mit der oben erwähnten allgemeinen Einschränkung) Rubners Daten den unsrigen gegenüberstellen, so bestehen bezüglich der Ausnutzung des Stickstoffes bei Ernährung mit Fleisch und hartgekochten Eiern keine wesentlichen Unterschiede, während nach unseren Versuchen der Stickstoff bei Ernährung mit weichgekochten und rohen Eiern besser ausgenutzt wird als bei Fleischernährung, wobei die rohen Eier nur unbedeutende Vorteile von den weichgekochten aufweisen. Bezüglich der Ausnutzung des Fettes aber kommt den hartgekochten Eiern eine im Verhältnis zum Fleische etwas erheblichere Resorptionsgröße als den weichgekochten und rohen Eiern zu. Wenn man aus den angeführten Versuchsresultaten Anwendungen für die praktische Diätetik gewinnen will, so wird man den weichgekochten und den rohen Eiern als dem Bestandteile einer gemischten Kost höheren Nährwert als dem Fleische zusprechen müssen. Dagegen findet die bei vielen Laien und auch manchen Aerzten bestehende starke Ueberschätzung gerade der rohen Eier in dem physiologischen Experiment keine ausreichende Begründung. In Anbetracht der guten Magenverdaulichkeit, ihres hohen Nährwertes und ihrer vorzüglichen Ausnutzbarkeit verdienen die Vogeleier (insbesondere die weichgekochten und rohen) eine ausgiebige Verwendung nicht nur in der Krankenkost, sondern auch in der eigentlichen Volksernährung.

Literatur: 1. Bull. of the Bureau of Labour No. 54 (September 1904). Hergestellt für die Weltausstellung in St. Louis. S. 1162. (Zitiert nach $\mathrm{C}_{0} \mathrm{~h} n \mathrm{he}$ im, Die Physiologie der Verdauung und Ernährung 1908, S. 456.) - 2. Uffelma n Deutsches Archiv für klinische Medizin 1877, Bd. 20. - 3. Penzold t, Deutsches Archlv für klinische Medizin 1893, Bd. 51. - 4. Prager, Ueber die Verdaulichkeit der für die Krankenernăhrung wichtigsten Spelsen und Getränke 1892 - 5. Rubner

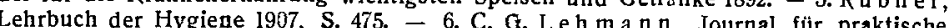
Chemie 1842, Bd. 27. - 7. Ru bu e r, Zeitschrift für Biologie 1879, Bd. 15. - 8. Le b bin Therapeutische Monatshefte 1901, No. 15 .
I) Frie d Iâ n de r, Ther. Monatshefte 1900 , S. 677; H e r m a n n, ebendaselbst, S. 12 und 33; Graul, Deutsche med. Wochenschr. 1901, S. 370; Ode nth a I, Deutsche zahnărztl. Wochenschr. 1901, No. 49. 